\title{
Epidemiology of intestinal parasitosis in Italy between 2005 and 2008: diagnostic techniques and methodologies
}

\author{
Daniele Crotti', Francesco Bernieri ${ }^{2}$, Annibale Raglio ${ }^{3}, \mathrm{AMCLI}$-CoSP ${ }^{4}$ Group Study $^{5}$ \\ I L. P. in Parassitologia, Perugia \\ 2 L. P. in Parassitologia, Milano \\ 3 USC Microbiologia e Virologia AO Ospedali Riuniti, Bergamo \\ 4 Associazione Microbiologi Clinici Italiani-AMCLI, Comitato di Studio AMCLI per la Parassitologia, Milano \\ 5 AMCLI-CoSP Group Study: elenco componenti in Appendice
}

Key words: Bowel parasitosis, Diagnosis, Epidemiology, Faeces, Helminths, O\&P, Protozoa

Epidemiologia delle parassitosi intestinali e tipologia delle tecniche adottate per la loro diagnosi in Italia nel periodo 2005 - 2008

\section{SUMMARY}

Aim of the study was to keep a real and actual photo relating to 2005-2008 regarding to diagnostic techniques and methodologies for intestinal parasites; so it would be possible to know specific epidemiology and suggest more rational and efficacious guide-lines.

All members of AMCLI were involved in the proposal of a retrospective study regarding bowel parasites, helminths and protozoa. To engaged laboratories we asked how O\&P was performed, if a specifical research for E. vermicularis and S. stercoralis was performed, if for the identification of $D$. fragilis, Entamoeba histolyticaldispar and Cryptosporidum spp were performed recommended specific permanent stains. 23 laboratories gave assent; but for an inferior number was possible to use the data for analysis and evaluation.

Relating O\&P only some laboratories performed permanent stains: Giemsa for D. fragilis, antigen and/or Trichrome stain for E. histolytica/dispar, antigen and/or acid fast stain for Cryptosporidium spp. Not all laboratories research specifically S. stercoralis. So the epidemiology is differentiated and related more to adequate or not adequate techniques than cohorts of examined populations.

The overall positivity for parasites ranged from $0 \%$ to $18.7 \%$, for protozoa (pathogens or not) were from $0 \%$ to $14.7 \%$; for nematodes from $0 \%$ to $3.7 \%$; for cestodes from $0 \%$ to $1.0 \%$; for trematodes from $0 \%$ to $1.0 \%$. Among helminths, E. vermicularis, followed by S. stercoralis, also in O\&P, is the most frequent. The specific research of S. stercoralis gave a positivity from $0 \%$ to $33.3 \%$; the cellophane tape test was positive for E. vermicularis from $0 \%$ fo $21.9 \%$ of cases.Among pathogen protozoa, D. fragilis, when permanent stain were applied, prevailed from $0 \%$ to $16.6 \%$; G. duodenalis from $0.8 \%$ to $4.3 \%$; E. histolytica/dispar, using a permanent stain or research of antigen, was identified from $0 \%$ to $20.6 \%$. Coccidia were very rare, with Cryptosporidium spp observed from $0 \%$ to $5.2 \%$.

These are our conclusions: many laboratories need for a critical revision of own technical procedures and diagnostic methodologies.

\section{INTRODUZIONE}

Nel corso del secolo attuale, nel nostro Paese, le problematiche concernenti le parassitosi intestinali, se da un lato sono passate in sordina, soprattutto per l'involuzione del sistema sanitario nazionale con conseguente carenza di risorse operative e diagnostiche al riguardo (personale sanitario e disponibilità economiche in primo piano), dall'altro i sia pur pochi lavori scientifici pubblicati su tale tema riflettono comunque l'interesse che ancora tali patologie suscitano $(1,5,18,19,22$, $23,25)$. Le parassitosi intestinali, siano esse causa di infezione intestinale acuta o cronica, siano esse causa di diarree, più spesso legate a infezioni protozoarie, o di patologie aspecifiche a livello del- l'apparato gastro-intestinale in senso lato inteso, solitamente espressione di infestazioni elmintiche, siano esse asintomatiche o apparentemente tali, sono tuttora presenti $(6,20,24,26,32,3)$. Le motivazioni sono tante e diversificate, e da tempo note: aspetti clinici e patologie di base (depressione immunitaria, terapie cortisoniche e altro ancora), viaggi, migrazioni ed immigrazioni, abitudini alimentari e condizioni igieniche, percorsi diagnostici (tecniche e metodologie) adeguati o insufficienti, idonei o non pertinenti, capacità e bontà diagnostiche strutturali ed individuali di chi opera in tale campo $(4,8,12,27,34)$.

Su tali considerazioni e sulla base di analoghe passate esperienze, dopo la metà del primo decen- 
nio del XXI secolo, il Comitato di Studio per la Parassitologia dell'Associazione Microbiologi Clinici Italiani, ha deciso di condurre una indagine epidemiologica, di tipo retrospettivo, relativa alle infezioni ed infestazioni parassitarie intestinali umane nel nostro Paese, focalizzando l'attenzione in particolar modo sulle tipologie diagnostiche adottate ed applicate $(2,3)$. I risultati di tale indagine, che coinvolse 23 differenti laboratori distribuiti lungo tutta la Penisola, furono presentati nel 2008 nel Corso del XXXVII Congresso Nazionale AMCLI, all'interno della Sessione PERCORSI DIAGNOSTICI, a Stresa (VB).

A distanza di quattro anni, abbiamo deciso di pubblicare i dati scaturiti da quella indagine policentrica, in quanto alcuni lavori scientifici pubblicati sulla rivista Microbiologia Medica e su altre riviste scientifiche italiane, hanno messo in risalto la necessità di portare a conoscenza $i$ limiti che ancora si osservano nella diagnostica parassitologica fecale, suggerendo così al contempo anche le migliori strategie operative specifiche, confermando l'importanza che le parassitosi intestinali ancora hanno in ambito medico, specialmente nel campo della sanità pubblica, parassitosi spesso se non quasi sempre di interesse anche veterinario, essendo buona parte di esse delle zoonosi, ovvero sia antropo-zoonosi o zoo-antroposi che dir si voglia $(9,10,30)$.

Le motivazioni, i quesiti, le problematiche che ci hanno spinto a tutto ciò sono facilmente spiegabili e comprensibili: quale e come può definirsi tale "l'esame copro-parassitologco standard" (ECPS)? L'ECPS è finalizzato alla ricerca di elminti e protozoi: quali e quanti? Quali altre tecniche possono e/o debbono essere impiegate o adottate in circostanze particolari? Quali sono i quadri clinici che possono sottendere un'infezione protozoaria e/o una infestazione elmintica? Quanti esami specifici fecali debbono essere condotti, ossia quanti campioni fecali o simili debbono essere analizzati per ciascun paziente o soggetto, per una buona riuscita dell'ECPS, ovvero per definire tale un ECPS? Queste principalmente le domande che ci spinsero allora a condurre l'indagine policentrica, ed ora a pubblicizzare i risultati, per meglio comprendere $\mathrm{i}$ limiti che molte pubblicazioni scientifiche ancora presentano a tale riguardo, per sollecitare una migliore impostazione diagnostica in tale campo, e come stimolo ad implementare le ricerche parassitologiche intestinali, per far sì che l'accreditamento possa essere raggiunto senza trascurare nulla, e quindi operare con conoscenza, coscienza, competenza e responsabilmente $(2,3,4,9,16)$.

L'indagine venne presentata con una lettera di accompagnamento mirata, come riportato in
Figura I. Lettera e questionario, di cui oltre, fu inviata a tutti i soci AMCLI dalla Segreteria Nazionale AMCLI utilizzando il servizio di posta elettronica, cui tutti o quasi i soci ne erano utilizzatori. La comunicazione fu inviata nelle primissime settimane del 2008.

\section{METODOLOGIA: MATERIALI E METODI}

All'inizio del 2008 da parte della Segreteria Nazionale AMCLI venne distribuita a pressoché tutti i soci iscritti all'Associazione Microbiologi Clinici Italiani, una scheda di tre pagine accompagnata da una lettera di accompagnamento, che spiegava in modo conciso l'intento dello studio. Tale scheda la possiamo suddividere in 3 parti.

La prima parte è quella che potremmo definire comune (Tabella 1a): viene richiesto dapprima di fornire i dati personali dell'operatore/compilatore del questionario e del responsabile per la Microbiologia/Parassitologia del singolo laboratorio diagnostico, sia esso ospedaliero, universitario o privato, chiedendo l'adesione o meno alla indagine. Se sì, si invitava anche a riportare l'anno di rilevazione dei dati o gli anni di tale rilevazione, relativi all'indagine in questione: $2005 \mathrm{e} / \mathrm{o}$ 2006 e/o 2007; eventualmente anche i dati dei primi sei mesi del 2008, data la scadenza stabilita inizialmente al 31 maggio ma poi procrastinata al 30 giugno. Sempre nella prima parte, quella comune o essenziale che dir si voglia, si invitava l'operatore a registrare le indagini eseguite ed i relativi risultati in riferimento al cosiddetto ESAME COPROPARASSITOLOGICO STANDARD (l'O\&P degli anglosassoni, ma che noi abbiamo deciso di chiamare con l'acronimo ECPS). In tale scheda il responsabile o chi per lui avrebbe dovuto registrare le indagini svolte, $\mathrm{i}$ risultati emersi, relativamente a elminti e protozoi, nonché le tecniche utilizzate (in Tabella 1 è riportato quanto veniva richiesto).

In Tabella $1 \mathrm{~b}$ si riporta la II parte della scheda inviata, che fa riferimento alla ricerca di due elminti, il cui reperimento con 1'ECPS è solitamente negativo o del tutto casuale: si chiedeva se il laboratorio eseguiva la ricerca mirata dell'ossiuro Enterobius vermicularis (come e quante volte) e la ricerca mirata di Strongyloides stercoralis (come e quante volte), fornendo ovviamente i risultati eventualmente scaturiti $(7,15)$.

In Tabella 1c si riporta invece la domanda che fu fatta relativamente a ricerche specifiche protozoarie, importantissime peraltro, ma che spesso sono disattese dai più comuni laboratori diagnostici.

In particolare si richiedeva se il laboratorio eseguiva specificatamente ed ad hoc la ricerca di Dientamoeba fragilis, ormai protozoo più frequente un po' in ogni dove nel mondo, che neces- 
sita di una specifica colorazione (come si dirà), con annessi e connessi (vedi Tabella relativa), se il laboratorio eseguiva la ricerca specifica di Cryptosporidum spp. (raro ma di indubbia importanza), e ovviamente come e su quanti campioni fecali, ed infine si richiedeva se il laboratorio ricercasse con colorazioni specifiche adeguate Entamoeba histolytica/dispar, l'una patogena, l'altra no, ma indistinguibili, in linea di massima, morfologicamente, l'una dall'altra, essendo non endemica in Italia la patogena (la prima delle due), ma presente la seconda così come presente la patogena vera e propria in soggetti immigrati (11).

Il presupposto di tutto ciò era ed è evidente.

Con l'ECPS senza microscopia la ricerca di una bella fetta di protozoi era latitante: amebe patogene vere e proprie (quanto meno le loro fasi trofozoitiche), il coccidio Cryptosporidium spp (ora Cryptosporidium hominis e C. parvum), e il flagellato atipico $D$. fragilis (seconda causa di diarrea acute dopo lo schizomicete Campylobacter spp nei paesi cosiddetti sviluppati, e anche in Italia, laddove le indagini sono o siano condotte correttamente in tutto e per tutto), non venivano ricercati e quindi refertati; la conseguenza era (ed è) una limitazione grave all'esame coproparassitologico, che così non può esser definito tale o standard (21).

Noi sappiamo ormai che le infezioni protozoarie hanno un rilevante peso anche nelle diarree acute, siano esse infantili che adulte, sia nel soggetto autoctono, che nel viaggiatore, sia nel soggetto immunocompetente che, a maggior ragione, in quello immunocompromesso. Mentre i coccidi Isospora (ora Cystoisospora) belli e Cyclospora caytanensis possono essere diagnosticate nel comune ECPS da occhio attento ed esperto, il piccolo coccidio Cryptosporidium (C. hominis, tipicamente umano, e C. parvum, agente zoonotico, sono morfologicamente simili se non uguali) necessita di una colorazione all'acido (alcool) - resistenza che non tutti sono in grado di eseguire (21).

Le problematiche relative agli elminti sono invece quelle già citate: l'ECPS non evidenzia tutti i patogeni parassitari (in senso di elminti, essenzialmente); E. vermicularis e S. stercoralis, per esempio, resterebbero esclusi nella stragrande maggioranza dei casi (i rari o meno casi sarebbero puramente da intendersi come reperti occasionali, casuali, fortuiti), a meno che non si ricorra a numerosi esami consimili che però avrebbero effetti non sempre favorevoli, anche in termini di costi e di eccessivi ritardi temporali $(2,3)$.

Le cose possono o potrebbero essere più complesse, e questo per garantire una ottima qualità diagnostica specifica; ma non sempre ciò è realizzabile, non sempre il beneficio paga il costo, non sempre i laboratori sono attrezzati per tutto ciò e per altre varianti o variabili diagnostiche, per rimanere sempre in campo di parassitosi intestinali (intese in senso ampio e lato), non sempre le richieste per la ricerca parassitologica sono chiaramente e correttamente espresse, sì da impostare nel migliore o comunque più ragionevole se non razionale dei modi tale indagine diagnostica di laboratorio.

Analizzeremo pertanto tutti i dati elaborati dei risultati pervenuti e indicheremo alla fine i limiti della medesima indagine e quanto ancora resta da fare, da applicare, da implementare, per qualificare, secondo i nostri schemi e in relazione a quanto sinora pubblicato al riguardo, la diagnostica parassitologica vera e propria. In altra sede, ma ora già pubblicati nel sito AMCLI, si possono consultare i PERCORSI DIAGNOSTICI UFFICIALI che l'AMCLI - COSP ha stabilito nel campo della parassitologia cosiddetta intestinale (2).

\section{RISULTATI}

In Tabella 2 riportiamo le risposte generiche pervenute. Una puntualizzazione è però necessaria. Arbitrariamente abbiamo cercato di suddividere i laboratori in 3 livelli: I, II e III. Al laboratorio di I livello competerebbe quanto esposto nella prima parte della Tabella 1 (Tabella 1a); a quello di II livello quanto esposto nella prima e nella seconda parte della medesima Tabella (Tabella 1b); al laboratorio di III livello competerebbe anche quanto riportato nella Tabella 3c. Ma non sempre così, crediamo, di fatto è poi avvenuto, né così probabilmente stanno le cose; ma tant'è.

Hanno risposto 25 strutture diagnostiche, di cui due non hanno fornito dati elaborabili. Pertanto l'analisi è sui dati (e non sempre, come si leggerà) di 23 laboratori complessivi, 17 al Nord Italia (Piemonte, Lombardia, Veneto, Trentino ed Emilia e Romagna), 2 nel Centro (Umbria e Lazio), e 4 al Sud (Campania e Sicilia). Si trattava di 22 strutture pubbliche (Ospedali e/o Policlinici) ed 1 struttura privata.

Per quanto riguarda le annate di riferimento dei dati rilevati e forniti va detto che:

- 1 laboratorio ha fornito i dati relativi al solo 2005; - 3 laboratori hanno fornito dati relativi al solo 2006; - 8 laboratori hanno fornito i dati relativi al solo 2007;

- 1 laboratorio ha fornito i dati relativi al biennio 2006 - 2007;

- 10 laboratori hanno fornito i dati relativi al periodo 2006 - 2007 - prima metà 2008.

In Tabella 3 si riportano le tecniche utilizzate per l'ECPS. Dodici laboratori (anche 5 di reputato III livello, date le ipotizzabili dimensioni della struttura ospedaliera) hanno eseguito soltanto un 
esame diretto con un arricchimento FEA (in un caso al FEA era sostituita la ben misera flottazione). Otto laboratori, soprattutto stimati di III livello, hanno eseguito correttamente oltre all'esame diretto e al FEA anche almeno 1 colorazione permanete (e riteniamo all'occorrenza). Soltanto 3 laboratori $(13 \%)$ hanno potuto eseguire un reale esame copro-parassitologico standard completo. In Tabella 4 sono riferiti il numero di esami eseguiti per anno dai vari Centri: il numero di esami è stato volutamente arrotondato per eccesso o difetto, sì da meglio o più facilmente interpretare i risultati. Come si può vedere, i dati sono estremamente vari nel numero: si passa dai 100 o dalle pochissime centinaia di alcuni laboratori di dimensioni contenute sino ai 4000, 5000 e 6000 circa di realtà decisamente più vaste. Anche se non sempre le cose sembrano o ci sarebbero parse scontate: da una struttura ospedaliera (e universitaria) come quelle di Palermo e di Roma, ma forse anche di Torino, ci saremmo aspettati un maggior numero di esami eseguiti e quindi di dati forniti; così come ci ha positivamente meravigliato $\mathrm{i}$ numeri elevati di strutture non grosse come Iseo, Gallarate e Montebelluna, per fare alcuni esempi. I perché potrebbero essere relazionati all'interesse anche personale che il microbiologo nutre verso tali problematiche parassitarie; o forse le cose stanno diversamente, ma allora non possiamo ipotizzare motivazioni e comprenderne il perché.

La Tabella 5 mostra con istogrammi la variabilità relativa al numero di campioni usualmente esaminati per soggetto. Se nel $9 \%$ non viene riferito, solamente nel $4 \%$ dei casi, e per fortuna, venne esaminato 1 solo campione, che come tutti ormai sanno, è spesso insufficiente. Nel $22 \%$ e $26 \%$ rispettivamente ad ogni soggetto erano esaminati almeno 2 campioni fecali o 3 . Nel 39\% invece vi era una discreta variabilità: da 1 a 3 . Noi sappiamo che il numero di campioni da analizzare va in linea di massima (enteriti acute a parte) da un minimo di 3 ad un massimo di 6 (per Giardia spp ed E. vermicularis: ne spiegheremo le motivazioni).

Ma vediamo le positività per parassiti complessive e specifiche che sono state osservate e segnalate dai singoli laboratori.

In Tabella 6 vengono riportate le positività complessive rilevate per patogeni e non patogeni all'ECPS. Le differenze sono enormi: dall' $0.5 \%$ di Palermo al $18.7 \%$ di Perugia. Ne analizzeremo le discrepanze. E non soltanto.

La Tabella 7 mette in evidenza le prevalenze percentuali rilevate per anno, su 17 laboratori: per quelli di Padova, Reggio Emilia, Gallarate (VA), Rho (MI), Montebelluna (TV), Chivasso (TO) non è stato possibile procedere data l'inadeguatezza o comunque incompletezza dei dati forniti.
Ciò ci rammarica. Lo ribadiremo nella discussione. I dati che sono riportati fanno fede a quanto operato nella singola struttura (vedi sopra); a dire che le grosse differenze percentuali sono legate, come più oltre si vedrà, al limite dell'ECPS stesso applicato (completo o ridotto, per essere facilmente comprensibili ma senza essere grossolani). Protozoi (patogeni o meno) repertati in quel di Perugia sono ben più alti di quelli ritrovati in tutti gli altri centri, fatte salve alcune realtà come Milano e Pavia, forse. Circa i nematodi, se le frequenze sono inevitabilmente sempre più basse rispetto ai protozoi, va segnalato come alcuni laboratori non ne abbiano mai segnalato alcuno, nonostante questo gruppo di elminti sia tuttora bene presente in Italia. Per cestodi e trematodi, i numeri sono ancora più bassi, soprattutto per i trematodi, quasi esclusivamente, questi ultimi, di importazione. In molti centri non sono stati mai segnalati.

Analizziamo nel dettaglio i singoli parassiti segnalati da tutti i centri nel loro insieme, cominciando dai tre gruppi di elminti. In Tabella 8 , il prospetto complessivo relativo all'insieme degli elminti vede in $S$. stercoralis il verme più spesso reperito, più frequente quindi, nell'ECPS, in Italia: 195 segnalazioni. I dati si riferiscono a 18 laboratori che parteciparono alla indagine. Segue E. vermicularis con 94 casi, Taenia spp con 91, e via via tutti gli altri. La tabella riporta i vari elminti in relazione alla frequenza di individuazione.

Per quanto riguarda i protozoi, su 17 laboratori che hanno fornito i dati, e dati utilizzabili per una loro analisi statistica, dalla Tabella 9 si evince come la stragrande maggioranza sia dovuta a protozoi non patogeni (pari a 1525), mentre tra gli altri protozoi, osservabili con certezza all'ECPS, Giardia duodenalis è stata identificata in 466 casi, Cystoisospora (Isospora) belli in 7 (casi di importazione), Cyclospora cayetanensis in 2 soli casi di importazione. I medesimi dati sono esposti in Tabella 10 utilizzando un modello statistico "a torta" per meglio inquadrare il dato complessivo emerso.

Questi i risultati scaturiti in riferimento all'ECPS.

Analizziamo ora le ricerche specifiche relative a E. vermicularis e a $S$. stercoralis, la cui biologia ed il ciclo vitale è tale per cui la ricerca specifica di tali nematodi è diversificata rispetto a tutti gli altri elminti.

Per quanto riguarda l'ossiuro E. vermicularis la sua ricerca venne dichiarata essere stata eseguita in 22 laboratori: in tutti i casi venne utilizzata la tecnica dello "scotch test" o "nastro adesivo". Il 27\% dei laboratori (6 laboratori) analizzò 1 solo 
campione per soggetto, il 50\% (11 laboratori) 2 o 3 campioni successivi, il 14\% (3 laboratori) da 1 a 3 o più campioni, mentre in 2 laboratori $(9 \%)$ non venne riferito. Il tutto è presentato in Tabella 11 . Nel paragrafo relativo alla discussione, cercheremo di analizzare queste enormi discrepanze e, se non giustificarle, quantomeno comprenderle o intuirle.

La ricerca delle larve di $S$. stercoralis deve essere condotta o con sistemi diversificati, come il Metodo di Baerman (o sue modifiche) o analoghi, oppure con la coltura delle medesime su agar specifico, oggi anche reperibile in commercio.

Così non facendo si hanno spesso sottostime, anche notevoli, salvo ricorrere, come in rarissimi Centri fanno, a numerose concentrazioni con FEA. Ma così operando si può poi correre il rischio di identificare come appartenenti a $S$. stercoralis larve di nematodi a vita libera. Intanto va detto che soltanto 12 laboratori hanno ricercato in modo mirato questo nematode particolarissimo. Un laboratorio (8\%) ha analizzato un solo campione, 7 laboratori $(60 \%)$ hanno analizzato 2 o 3 campioni, 2 laboratori (16\%) hanno riferito di aver analizzato un numero variabile di campioni, 1 solo laboratorio ( $8 \%$ ) ha analizzato oltre 3 campioni, mentre un ultimo laboratorio $(8 \%)$ non ha riferito nulla al riguardo (in Tabella 12 un istogramma rappresenta tutto ciò).

Per quanto concerne poi il metodo utilizzato allo scopo, ecco i risultati: 3 laboratori (25\%) hanno utilizzato il Metodo di Baerman o sue modifiche, 5 laboratori $(42 \%)$ hanno impiegato la coltura su agar, 4 laboratori (33\%) hanno alternativamente utilizzato l'uno e/o l'altro.

I risultati sono riportati in Tabella 13. In 4 Centri, sia pur in pochissimi casi, $S$. stercoralis non è mai stato reperito, mentre le percentuali negli altri 8 le percentuali sono variate dal $2.2 \%$ al $33.3 \%$, ma con numeri su cui dobbiamo fare alcune considerazioni nel paragrafo successivo.

\section{Veniamo ai protozoi}

La ricerca mirata di $D$. fragilis è stata condotta in soli 7 laboratori. La ricerca per una diagnosi di certezza deve essere condotta con una colorazione permanente che prevede fasi preanalitiche diversificate; tale colorazione può essere la colorazione secondo Giemsa (con un paio di varianti), la colorazione cosiddetta Tricromica, la colorazione all'Ematossilina - ferrica, anche se altri Autori, non nel nostro Paese, ne possono utilizzare altre ancora. In Tabella 14 si riporta tutto questo con i risultati ottenuti. In un centro, su 10 soggetti analizzati, tale protozoo non è mai stato reperito, mentre negli altri 6 le percentuali sono variate dallo $0.9 \%$ al $16.6 \%$, anche se in quest'ultimo caso le ricerche furono talmente rare ed evidentemente selezionate per cui il dato qual tale andrebbe preso con le pinze, come si dice. Sempre nella Tabella 14 vengono riportate, a confronto, anche le positività relative a $G$. duodenalis, ricerche effettuate solitamente con il comune ECPS (esame a fresco e arricchimento/concentrazione FEA). I due protozoi sono entrambi dei flagellati ma hanno peculiarità biologiche del tutto diverse, laddove il quadro clinico è sovente sovrapponibile. Abbiamo riportato tale confronto, per sottolineare quanto da tempo e da tanti si dice, che $D$. fragilis è spesso e volentieri più frequente della ben più conosciuta G. duodenalis (o G. lamblia, o G. intestinalis). Così anche in questa nostra indagine. Nel paragrafo successivo esamineremo il quanto.

La ricerca specifica di Cryptosporidium spp è stata eseguita in 16 laboratori e quasi sempre soltanto su richiesta specifica; in Tabella 16 si riportano i risultati. In tutti i casi è sempre stata eseguita la colorazione di Ziehl-Neelsen (ZN) modificata; nel centro di Pavia e di Perugia questa era preceduta da una colorazione estemporanea ad hoc, nel centro di Torino, di Milano, di Perugia e di Napoli era talora eseguita anche l'immunofluorescenza diretta specifica, utilizzando kit commerciali. In 8 laboratori tale coccidio non è mai stato reperito; negli altri 8 le prevalenze sono state tra lo $0.4 \%$ e il $5.2 \%$ (vedi sempre tabella 16 ).

La ricerca di certezza e mirata di E. histolytica / E. dispar (cisti e/o trofozoiti) è stata condotta, quando richiesto o ritenuto opportuno, da parte di soli 8 laboratori diagnostici, essenzialmente in realtà importanti. I risultati sono riportati in Tabella 17. In 2 casi mai fu reperita. Negli altri casi le percentuali sono variate dallo $0.2 \%$ al $20.6 \%$. Va precisato che i sistemi utilizzati (vedi medesima Tabella 17) mettevano in evidenza il binomio $E$. histolytica / E. dispar, la prima patogena, la seconda non patogena. La morfologia delle due amebe è uguale, salvo casi eccezionali, per cui tale non può che essere la refertazione. Ne riparleremo oltre.

\section{DISCUSSIONE}

Nonostante la buona diffusione dell'informazione relativa a codesta indagine policentrica, peraltro da tempo da molti sollecitata, la risposta dei laboratori diagnostici è stata piuttosto bassa, inferiore a quanto ci saremmo aspettati. È anche vero, pur tuttavia, che è probabile che molti laboratori non abbiano risposto o perché le indagini parassitologiche non vengono o vengono di rado eseguite o in quanto la possibile scarsità dei dati o dei risultati positivi ha influito sulla decisione di partecipare e quindi rispondere come raccomandato. 
Va inoltre detto come alcuni laboratori abbiano male interpretato le indicazioni, e quindi abbiano fornito dati, forse anche interessanti, ma non utilizzabili per potere essere analizzati.

Per quanto riguarda le indagini coproparassitologiche va sottolineato, come osservabile in Tabella 3 , che ben pochi laboratori siano in grado di eseguire un corretto e completo esame parassitologico delle feci, o comunque dei campioni fecali. Vi è decisamente una carenza e una refrattarietà a eseguire almeno una colorazione permanente, necessaria per la sicurezza di identificare $D$. fragilis nonché quella di potere con certezza identificare le amebe sia nel loro genere che nella specie di appartenenza. Il FEA viene comunque eseguito da tutti i laboratori che hanno risposto, tranne uno che ancora si limita a eseguire l'inadeguato, in campo umano, esame per semplice flottazione (non è stato riferito quale tipo).

Va altresì ancora ribadito che male si comportano quei laboratori diagnostici che operano su un solo campioni fecale, o anche su due campioni; l'ECPS va eseguito sempre su almeno 3 campioni fecali, spontaneamente emessi, e a giorni preferibilmente alternati, in quanto non sempre cisti e/o uova di parassiti vengono espulse. Le cose possono cambiare se si è in presenza di una enterite acuta, nel qual caso, assieme al necessario esame microbiologico delle feci, una sola ricerca parassitologica, meglio però con una colorazione permanente (Giemsa o Tricromica o altra), può bastare. Va peraltro ricordato come in caso di sospetta giardiasi, può essere necessario ricorrere sino a 6 campionamenti fecali, laddove i precedenti risultassero negativi, per essere sicuri di escludere o individuare G. duodenalis; questo protozoo colonizza ed infetta il duodeno, ove vive in fase trofozoitica; la sua trasformazione in cisti avviene nella discesa lungo il colon e le cisti medesime vengono eliminate irregolarmente con il materiale fecale (quando l'infezione è asintomatica o aspecifica e le feci sono solide).

Come indicato nel Percorso diagnostico reperibile nel sito dell'AMCLI, è utile ricordare:

1) la necessità e utilità di acquisire informazioni cliniche ed anamnestiche;

2) se non si riesce ad applicare su tutti i campioni l'ECPS corretto (FEA e colorazione permanente Giemsa o Tricromica), è utile, in base alle informazioni clinico-anamnestiche e/o al tipo di campione inviato (formato o liquido), proporre un approfondimento diagnostico per esempio per D. fragilis oppure per E. histolytica o per S. stercoralis;

3) per alcuni patogeni, schistosomi ed E. histolyti$c a$, può essere utile anche un approfondimento con indagini sierologiche.
La variabilità, anche elevata, delle frequenze numeriche complessive e percentuale del reperimento di parassiti nel loro complesso, è così, presumibilmente, da imputarsi al differente approccio diagnostico, ovvero sia a numeri più bassi corrispondono indagini meno complesse, meno complete, insufficienti e/o inadeguate.

Per quanto riguarda le positività osservate, riteniamo che quanto emerso altro non fa che confermare quanto da tempo in Itala segnalato, indipendentemente dal fatto che i soggetti analizzati fossero autoctoni o provenienti da aree a ben maggiore endemia. I protozoi sono prevalenti, soprattutto perché numerosi sono i non patogeni; tra gli elminti se più frequenti risultano essere i nematodi, anche perché, alcuni di essi, indubbiamente ancora presenti in Italia, i trematodi sono decisamente i più rari e soprattutto sono di importazione, con qualche eccezione, e si fa riferimento a Opisthorchis felineus $(13,28)$.

Sempre rimanendo in ambito di elminti, sia pur con l'insufficiente ECPS, S. stercoralis ed E. vermicularis sono sicuramente i più frequenti e tuttora presenti nella nostra Penisola, sia nell'uomo sia evidentemente nell'ambiente terreno più idoneo (clima temperato e ambiente umido).

Se Hymenolepis nana è riportata meno frequentemente di quanto attendibile, Taenia spp (di fatto $T$. saginata) è meno di rado reperita, sia pur con frequenze assai diverse nei vari centri ove segnalata; questo è forse legato alle abitudini umane e alle condizioni igieniche diversificate $(31,35,37)$. Nella lettura della Tabella 8 , va da sé immaginare il reperimento delle uova di alcuni elminti esclusivamente in soggetti immigrati, in quanto tale parassitosi in Italia non vi sono più, o mai vi sono state (di fatto però le sole schistosomiasi e capillariasi).

Nelle Tabelle 11 e 12 vengono riportati i risultati relativi alla ricerca mirata di $E$. vermicularis; nelle Tabelle 13 e 14 quelli relativi a $S$. stercoralis. Sono necessarie alcune fondamentali considerazioni. Innanzitutto va ribadito che anche per gli ossiuri vanno eseguiti almeno 3 campioni (scotch test o test del nastro adesivo, a nostro avviso migliore del tampone anorettale); per escluderlo con certezza va poi detto che può essere necessario ricorrere anche a 6 campioni e questo in quanto il punto debole di questo esame è il momento preanalitico della sua raccolta, della sua esecuzione: il bambino (E. vermicularis è nettamente più frequente in bambini in età scolare che in altri soggetti) spesso non può collaborare, piange, si divincola, e il genitore (spesso è proprio il genitore a raccogliere il campione) male esegue il campionamento! In ogni caso, così operando, E. vermicularis è nettamente il parassita in assoluto più frequente di tutti; ma questo dato era atteso. 
Le frequenze (vedi tabella 12), variabili da pochi a numerosi casi, da frequenza basse a frequenze non certo basse, sono legate un po' a tutte queste variabili, ovvero popolazione indagata, numero di esami eseguiti per soggetto o paziente, caratteristiche oggettive legate all'ambiente (scolastico o meno) più che allo stato sociale o alla provenienza dei soggetti, usualmente pediatrici.

In tema di strongyloidiasi, valgono analoghe considerazioni come per le enterobiasi. Se cercato con dovizia, la sua frequenza cresce, a maggior ragione se il metodo prescelto (purché raccomandato perché più adeguato) è utilizzato su più di un solo campione. Le diverse frequenze di reperimento (vedi Tabella 13 e 14) sono da attribuire alle solite variabili, ma qui sicuramente la variabile legata alla realtà geografica ambientale gioca un ruolo assai importante. In ogni caso, S. stercoralis resta il secondo elminta più frequentemente reperito, soprattutto quando cercato ad hoc.

Come esposto in Tabella 9 ed in Tabella 10, G. duodenalis è nettamente il più frequente protozoo, tra i patogeni, osservabile nell'ECPS, qualora non si esegua anche una colorazione permanente idonea e quella specifica per Cryptosporidium spp, come si dirà più avanti. Numerosi sono invece $\mathrm{i}$ protozoi non patogeni. Tra questi prevale Blastocystis hominis (reperita in circa i $2 / 3$ dei casi di tutti i non patogeni), e a seguire Entamoeba coli, Endolimax nana e altre più raramente.

Non ne riportiamo nel dettaglio le prevalenze, numeriche e percentuali, e avendo interpretato come saprofita non patogeno la stessa $B$. hominis, oggi giorno da alcuni forse rivalutata (38). In ogni caso è nostra opinione, se non convinzione, che il reperimento di parassiti, nella fattispecie protozoi, non patogeni, vada comunque refertato, in quanto espressione o di un danno, in ogni caso, in sede intestinale (e quindi spia di una patologia da meglio individuare e diagnosticare), o di possibili situazioni sanitarie e/o alimentari non adeguate né consone alla realtà più comune, quanto meno nel nostro Paese. Va anche poi detto che la presenza di protozoi non patogeni, oltre a essere spia di ciò, possono essere considerati opportunisti, in senso patologico, a dire che accanto ad essi altri veri patogeni vanno ricercati adeguatamente e approfonditamente prima di escluderli (da qui la ripetizione, anche più volte, dell'ECPS).

I coccidi, rari e di importazione, non prendono qui in considerazione Cryptosporidium spp. per il quale la ricerca deve essere mirata. Laddove e se ricercato (Tabella 16), è presente, con frequenze sempre piuttosto basse, ma più alte degli altri coccidi, e osservabile soprattutto in bambini e/o in soggetti / pazienti immunodepressi.

Le maggiormente dolenti note appaiono allorché si esamini la problematica relativa soprattutto se non esclusivamente alla ricerca di $D$. fragilis. Oramai riconosciuto, oltreché patogeno o potenzialmente tale (ma questo potrebbe valere anche per altri parassiti, Giardia spp in primis), anche come presente, e sovente, sia in paesi tecnologicamente avanzati che in quelli in via di sviluppo tecnologico, questo protozoo del tutto particolare è emerso predominante, tra i protozoi, anche in questo studio, sebbene pochi siano stati i laboratori diagnostici attrezzati al riguardo. Ad ogni buon conto, quando cercata secondo le indicazioni specifiche, D. fragilis, tranne che in un caso o due (Tabella 15), è sempre apparsa come la più frequente tra i patogeni e quindi più frequente di $G$. duodenalis. Le variate frequenze sono forse da imputarsi ancora una volta, non tanto alle differenti endemie, quanto alla capacità e bontà diagnostiche dei rispettivi laboratori $(11,14)$.

Per completare la discussione in ambito protozoario, soffermiamoci sulla Tabella 17 , relativa alle colorazioni specifiche per la identificazione corretta e certa delle amebe vere e proprie, sia nel genere, come detto, che nella specie di appartenenza. Ben pochi laboratori sono tuttora attrezzati per la loro identificazione, identificazione che necessita o della colorazione tricromica o in alternativa quella all'ematossilina ferrica (più che al Giemsa che malissimo inquadra le caratteristiche dei nuclei) oppure della esecuzione di tecniche biomolecolari, come il Triage (riferito da alcuni laboratori), metodo commerciale (come altri, ma in questo studio non riferiti) di facile utilizzo (che peraltro prevederebbe una conferma con colorazione adeguata), utile anche per altri protozoi (analoghe considerazioni al riguardo). Nella diagnostica routinaria non è possibile distinguere la patogena $E$. histolytica vera e propria dalla non patogena $E$. dispar, del tutto simile alla precedente. Soltanto in presenza di un quadro clinico eclatante con il rinvenimento di trofozoiti che abbiano fagocitato emazie, si potrebbe ipotizzare la forma patogena a discapito della non patogena.

Ma senza entrare nel merito specialistico (andrebbero eseguite colture specifiche e successive diagnosi particolari non certo alla portata dei comuni centri diagnostici), vale forse la pena rammentare come soltanto con una colorazione specifica è possibile identificare i trofozoiti amebici; l'ECPS permette invece e soltanto, e non sempre peraltro, la identificazione delle cisti amebiche, e di solito di quelle non patogene, che hanno un diverso significato, come sopra riferito. In questo studio, anche per E. histolytica/dispar valgono le medesime considerazioni fatte per la simil-ameba $D$. fragilis: la differente frequenza di reperimento è più legata al tipo di paziente e al tipo di approccio dia- 
gnostico (anche metodologico) utilizzato ed applicato, che non a realtà geografiche, fermo restando il fatto che E. histolytica è da considerarsi di fatto ed esclusivamente di importazione (da Paesi endemici, paesi cosiddetti in via di sviluppo, brutto termine ma ormai consolidatosi nel tempo e nell'uso del suo utilizzo).

Portiamo a termine la discussione segnalando alcune curiosità: 2 ancilostomidi (su 16) sono stati isolati in coltura (dato comprensibilissimo); nella realtà napoletana sembra essere elevata la prevalenza, tra i protozoi non patogeni, di Enteromonas hominis (non siamo in grado di esprimere un giudizio al riguardo); Taenia spp è stata soprattutto reperita, quindi è relativamente frequente, a Torino e a Napoli (due realtà a parer nostro apparentemente diverse); la segnalazione dei casi di Dicrocoelium dendriticum sono tutti casi di reperimento di uova di transito passivo (29); è stato segnalato infine 1 caso, prima non riportato, di Dipylidium caninum a Reggio Emilia (ma non possiamo confermarlo).

\section{CONCLUSIONI}

Potremmo dilungarci ancora nel riassumere le conclusioni che da questo studio sono emerse. Ma ci ripeteremmo, probabilmente.

Le parassitosi intestinali sono presenti, in Italia come altrove, in soggetti autoctoni come in viaggiatori, migranti ed immigrati, in bambini ed in adulti, con ragionevoli differenze legate, più che al sesso, all'età e alle abitudini legate a svariati fattori (ambientali, sociali, scolastici, famigliari, individuali). È doveroso condurre le indagini parassitologiche spesso, forse più frequentemente di quanto ipotizzabile. Certo è che una loro ricerca deve essere mirata e razionale: ECPS completo con l'aggiunta di altre indagini qualora si sospetti una diversificata parassitosi $(2,3,4,6,36)$.

Siamo coscienti che non tutti o forse pochi laboratori possano essere attrezzati a procedere in tale direzione. Ma forse allora sarebbe preferibile evitare di eseguire tali tipi di indagini, una tantum e in maniera inadeguata e/o insufficiente, demandando a strutture più complete la loro esecuzione, fermo restando che, quando eseguite, vengano dichiarati i limiti delle medesime, sì che l'utente ed il rispettivo medico curante possa o possano provvedere alla loro valutazione e quindi alla ripetizione in sedi più congrue e più garantiste di qualità e bontà diagnostiche.

L'AMCLI - CoSP, ovverosia il Comitato di Studio per la Parassitologia dell'Associazione dei Microbiologi Clinici Italiani, ha da tempo e in più occasioni sottolineato tutto ciò, fornendo al contempo linee guide operative e proponendo percorsi diagnostici più adeguati, finalizzati, e razionali al riguardo. $\mathrm{Ci}$ auguriamo che vengano così fatti propri da chiunque operi in tale settore, ed applicati nei modi e nei tempi raccomandati. Soltanto così

Figura I. Lettera di accompagnamento distribuita ai Soci AMCLI per aderire all'Indagine Policentrica Retrospettiva organizzata dall'AMCLI-COSP nel 2007-2008

\section{AMCLI -COSP \\ ASSOCIAZIONE MICROBIOLOGI CLINICI ITALIANI \\ Comitato di Studio per la Parassitologia \\ Indagine nazionale retrospettiva sulla diffusione delle parassitosi intestinali}

II Comitato di Studio per la Parassitologia dell'AMCLI, a distanza di oltre un decennio, propone una nuova indagine nazionale a carattere retrospettivo sulla diffusione delle parassitosi intestinali.

La finalità dell'indagine è quella di valutare l'operatività in tale campo della Parassitologia diagnostica (e più in generale della Microbiologia Clinica) onde verificare quali e quante possano essere tali parassitosi nel nostro Paese, sia autoctone che importate, e al contempo fornire preziose indicazioni atte a stilare un nuovo Nomenclatore Nazionale tale da favorire una maggiore e migliore qualità alle prestazioni analitiche nei laboratori microbiologici. Per tale Nomenclatore l'AMCLI e il CoSP stanno da tempo lavorando; i risultati di tali lavori e di tale indagine, se gli obiettivi saranno perseguiti, anche con l'apporto di molti, se non di tutti, verranno presentati al prossimo Congresso Nazionale AMCLI del 2008.

Speriamo che tutti possano partecipare favorendo in tempi reali i dati richiesti. Onde facilitare il compito, in tale indagine ci limitiamo a poche domande essenziali, tramite un adeguato questionario, cui tutti gli aderenti dovranno rispondere in modo esaustivo, corretto e responsabile.

Proponiamo così almeno un anno di osservazione: il 2007, e/o il 2006, e/o il 2005, in virtù della possibilità da parte di tutti di elaborare, se già non fatto, i propri dati e risultati.

Tutte le schede dovranno essere inviate, entro il 31 maggio 2008. 
facendo ed operando, si potranno colmare quelle lacune diagnostiche (oltreché epidemiologiche) e garantire al meglio un servizio medico-sanitario per il quale siamo stati preposti $(2,3,4,8,9,16)$.

\section{Appendice: Componenti gruppo di studio cor- relato al CoSP-AMCLI}

Mirella De Paola ed Elisa Burdino di Torino, Rosella Bruno di Chivasso (TO), Giovanna Castagna di Piancavallo (VB), Carla Sturla di Gallarate (VA), Romualdo Grande di Milano, Marina De Re di Rho (MI), Giuseppina Rocca di Binasco (MI), Simonetta Gatti di Pavia, Eugenio
Sala di Como, Fernanda Lanzini di Iseo (BG), Manuela Pegoraro di Verona, Valeria Besutti ed Ettore De Canale di Padova, Manuela Mistretta di Negrar (VR), Luciano Vettorato di Montebelluna (TV), Manuela Angeli e Vincenzo Cutrupi di Tione (TN), Carlotta Guidetti e Luigi Vecchia di Reggio Emilia, Massimo Confalonieri e M. Milanda Bertelli di Piacenza, M. Letizia D'Annibale di Perugia, Giulia Gilardi di Roma, Silvana Piccoli e Fabio Rossano di Napoli, Dino De Conno di S. Bartolomeo in Galdo (BN), Ildebrando Patamia di Catania, M. Teresa Baldi di Palermo.

Tabella Ia. Questionario: I parte (vedi testo).

$$
\begin{aligned}
& \text { AMCLI - CoSP } \\
& \begin{array}{l}
\text { Indagine nazionale retrospettiva sulla diffusione delle } \\
\text { Parassitosi intestinali }
\end{array}
\end{aligned}
$$

Laboratorio:

Struttura di appartenenza:

Città: ; Provincia: ; Regione:

Compilatore questionario:

Responsabile per la Microbiologia/Parassitologia:

Direttore: Aderisco all'indagine: $\square$ SI $\square$ NO

Anno/i di rilevazione: $\square$ I semestre 2008

$\square 2007$

$\square 2006$

ESAME COPROPARASSITOLOGICO STANDARD $($ ECPS $=0 \& P)$

(finalizzato alla ricerca di elminti e parte dei protozoi)

$\begin{array}{ll}\text { Indagini eseguite: } & \text { Risultati positivi: } \\ \text { Soggetti esaminati: } & \text { Soggetti parassitati: }\end{array}$

Numero di campioni analizzati per soggetto: $\square 1 \quad \square 2 \quad \square 3 \quad \square$ più di 3

Tecnica/e utilizzata/e: $\square$ microscopia diretta e dopo arricchimento formolo-etere/etilacetato colorazione/i estemporanea/e: colorazione/i permanente/i:

$\square$ altro:

POSITIVITA':

Elminti:

(indicare se uova e/o larve e/o altro)

Protozoi:

(indicare se cisti, oocisti, trofozoiti) 
Tabella Ib. Questionario: Il parte (vedi testo).

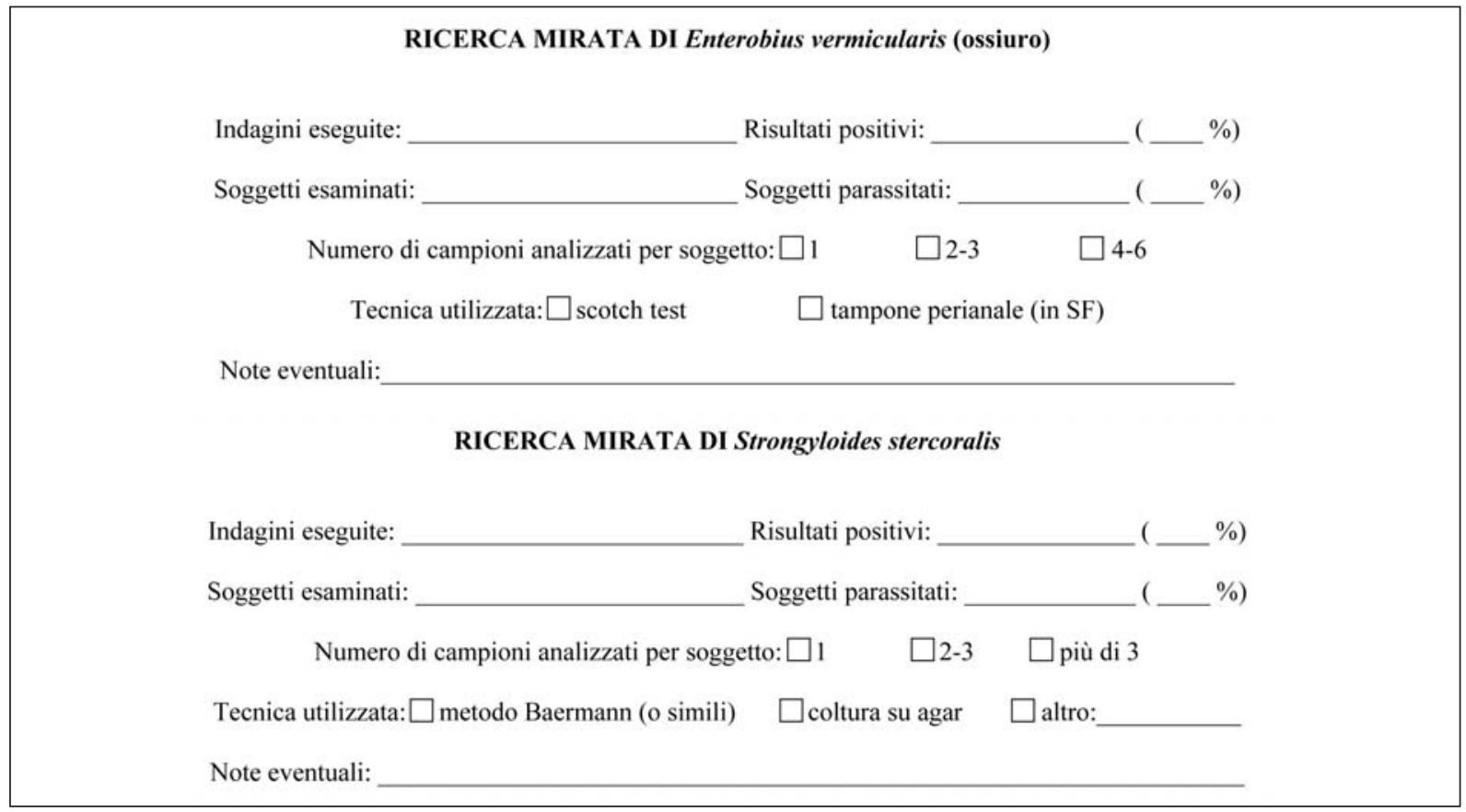

Tabella I c. Questionario: III parte (vedi testo).

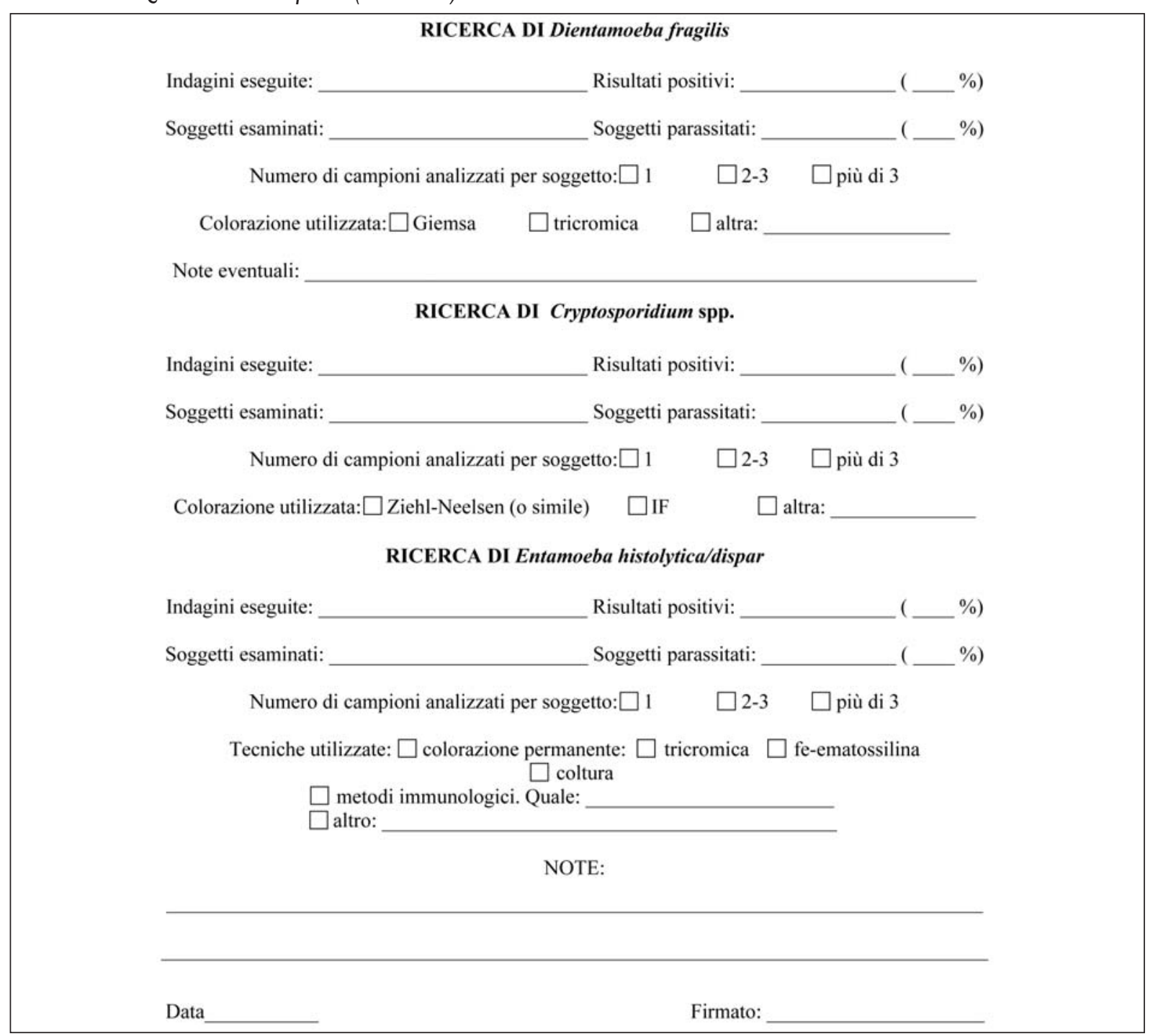


Tabella 2. Risposte pervenute allinvio del questionario.

Questionario:

\begin{tabular}{lcccc}
\hline Laboratorio & Nord & Centro & Sud & Totali \\
\hline III livello & 8 & 2 & 3 & 13 \\
\hline II livello & 5 & - & - & 5 \\
\hline I livello & 4 & - & 1 & 5 \\
\hline Complessivi & 17 & & 4 & 23
\end{tabular}

Note:

- I laboratorio (II livello) ha risposto senza potere fornire dati

- I laboratorio (III livello) ha risposto senza fornire dati

- Regioni coinvolte: Piemonte, Lombardia,Veneto, Trentino, Emilia-Romagna, Umbria, Lazio, Campania, Sicilia

- I laboratorio privato e 22 (24) strutture pubbliche

Tabella 3. Tecniche impiegate nell'esame copro - parassitologico da parte dei centri che hanno aderito all'indagine nazionale. Esame coproparassitologico standard (ECPS = O\&P)

\begin{tabular}{lcccc}
\hline Tecniche impiegate & Lab III & Lab II & Lab I & complessivi \\
\hline Esame diretto + FEA & & & & \\
+ I colorazione permanente + altro & 3 & & & 3 \\
\hline Esame diretto + FEA & & & 2 & 8 \\
+ almeno I colorazione permanente & 5 & I & 2 & II \\
\hline Esame diretto + FEA & 5 & 4 & I & I \\
\hline Esame diretto + flottazione & & $\mathbf{5}$ & $\mathbf{5}$ \\
\hline TOTALI & $\mathbf{I 3}$ & & & \\
\hline
\end{tabular}

Note: Lab III, II, I = laboratorio di III, II, I livello

FEA: arricchimento secondo concentrazione formolo - etere/etilacetato

Tabella 4. Prospetto degli esami eseguiti dai centri per anno.

\begin{tabular}{|c|c|c|}
\hline \multicolumn{3}{|c|}{ ECPS: $n^{\circ}$ di esami eseguiti per anno (arrotondati per eccesso o difetto) } \\
\hline & & I500 Gallarate (VA), Ospedale \\
\hline 100 & S. Bartolomeo in Galdo (BN) & I500 Napoli, Ospedale \\
\hline 150 & Tione (TN), Ospedale & 2000 Torino 2, Ospedale \\
\hline 200 & Piancavallo (VB), Ospedale & 2000 Como, Ospedale \\
\hline 350 & Binasco (MI), Ospedale & 2000 Reggio Emilia, Ospedale \\
\hline 500 & Palermo, Ospedale & 2000 Milano, Ospedale \\
\hline 500 & Roma, Ospedale & 2000 Bergamo, Ospedale \\
\hline 650 & Torino I, Ospedale & 2000 Montebelluna (TV), Ospadale \\
\hline 1000 & Iseo (BS), Ospedale & 2000 Verona, Ospedale \\
\hline 1000 & Chivasso (TO), Ospedale & 4000 Perugia, Ospedale \\
\hline 1000 & Rho (MI), Ospedale & 5000 Pavia, Ospedale \\
\hline 1000 & Catania, Ospedale & 6000 Padova, Ospedale \\
\hline
\end{tabular}

Tabella 5 .

Prospetto in diagramma del numero di campioni usualmente esaminati per soggetto.

\section{NUMERO DI CAMPIONI USUALMENTE ESAMINATI PER SOGGETTO}

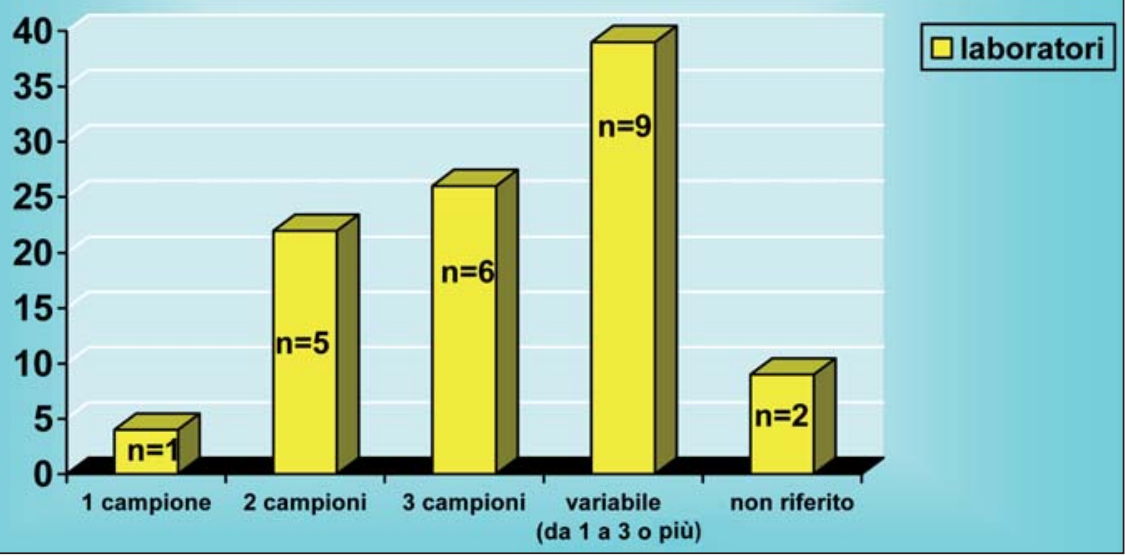


Tabella 6. Prospetto risultati generali: positività complessivo osservate centro per centro, in ordine decrescente di positività reperite.

\begin{tabular}{llrl}
\hline & ECPS: positività complessive rilevate (patogeni e non patogeni) \\
\hline $18.7 \%$ & Perugia & $3.3 \%$ & S. Bartolomeo in Galdo (BN) \\
\hline $10.1 \%$ & Pavia & $3.3 \%$ & Bergamo \\
\hline $8.8 \%$ & Padova & $2.6 \%$ & Torino 2 \\
\hline $8.7 \%$ & Catania & $2.0 \%$ & Gallarate (VA) \\
\hline $8.4 \%$ & Milano & $1.5 \%$ & Rho (MI) \\
\hline $7.0 \%$ & Napoli & $1.3 \%$ & Chivasso (TO) \\
\hline $6.7 \%$ & Iseo (BS) & $1.2 \%$ & Como \\
\hline $5.9 \%$ & Verona & $0.8 \%$ & Binasco (MI) \\
\hline $5.0 \%$ & Roma & $0.5 \%$ & Palermo \\
\hline $4.1 \%$ & Reggio Emilia & - & Montebelluna (TV) \\
\hline $3.4 \%$ & Torino I & - & Piancavallo (VB) \\
\hline $3.4 \%$ & Tione (TN) & \\
\hline
\end{tabular}

Tabella 7. Prevalenze percentuali complessive osservate in 17 laboratori che hanno fornito i dati in maniera corretta ed esaustiva (nei limiti della qualità delle scelte operate): PROTOZOI ed ELMINTI.

\begin{tabular}{|c|c|c|c|c|}
\hline \multicolumn{5}{|c|}{ ESAME COPROPARASSITOLOGICO (STANDARD) } \\
\hline & PROTOZOI & NEMATODI & CESTODI & TREMATODI \\
\hline Perugia & 14.7 & 2.5 & 0.5 & 1.0 \\
\hline Verona & 4.4 & I.I & 0.4 & - \\
\hline Bergamo & 1.7 & 0.5 & 0.7 & 0.2 \\
\hline Catania & 7.6 & 0.5 & 0.7 & - \\
\hline Torino I & 2.9 & I & $\mathrm{I}$ & - \\
\hline Roma & 5.9 & 3 & - & 0.6 \\
\hline Napoli & 8.4 & 0.4 & 0.3 & - \\
\hline Milano & 11.8 & 1.4 & 0.4 & 0.3 \\
\hline Palermo & 0.5 & - & 0.3 & - \\
\hline Como & $\mathrm{I}$ & - & 0.2 & - \\
\hline Pavia & 9 & 3.7 & 0.8 & 0.2 \\
\hline Torino 2 & 2 & 0.3 & 0.3 & - \\
\hline Tione (TN) & 0.3 & 2.9 & 0.3 & - \\
\hline Binasco (MI) & 0.7 & 0.7 & - & - \\
\hline Iseo (BS) & 6.6 & 0.6 & 0.5 & - \\
\hline S. B. in Galdo (BN) & 3.2 & - & - & - \\
\hline Piancavallo (VB) & - & - & - & - \\
\hline Mediana stimata & 3.5 & 1.1 & 0.4 & - \\
\hline
\end{tabular}

Non quantificabili: Reggio Emilia, Gallarate (VA), Rho (MI), Montebelluna (TV), Chivasso (TO)

Tabella 8. Numero di elminti reperiti, in ordine di frequenza (indipendentemente se autoctoni o di importazione)

\begin{tabular}{lc}
\hline \multicolumn{1}{c}{ ECPS: elminti reperiti e segnalati (in I8 laboratori) } \\
\hline Strongyloides stercoralis & 195 \\
\hline Enterobius vermicularis & 94 \\
\hline Tenia saginata I Taenia solium & 91 \\
\hline Ascaris lumbricoides & 41 \\
\hline Trichuris trichiura & 40 \\
\hline Hymenolepis nana & 36 \\
\hline Schistosoma mansoni & 30
\end{tabular}

\begin{tabular}{ll}
\hline Ancilostomidi & 16 \\
\hline Diphyllobothrium latum & 12 \\
\hline
\end{tabular}

\begin{tabular}{ll}
\hline Opisthorchis felineus & 8 \\
\hline Dicocoelium dendriticum & 6 \\
\hline Opisthorchis / Clonorchis spp & 2
\end{tabular}

\begin{tabular}{ll}
\hline Capillaria philippinensis & $\mathrm{I}$ \\
\hline Trichostrongylus spp. & $\mathrm{I}$ \\
\hline Hymenolepis diminuta & $\mathrm{I}$ \\
\hline Schistosoma japonicum & $\mathrm{I}$
\end{tabular}


Tabella 9. Generi e specie di protozoi reperiti al comune ECPS da 17 laboratori

\begin{tabular}{lc}
\hline \multicolumn{2}{c}{ Numero assoluto di PROTOZOI reperiti e segnalati (in I 7 laboratori) } \\
\hline Giardia duodenalis & 466 \\
\hline Isospora belli (ora Cystoisospora belli) & 7 \\
\hline Cyclospora cayetanensis & 2 \\
\hline Protozoi non patogeni & 1.525 \\
\hline
\end{tabular}

Tabella 10.

Prospetto a torta dei protozoi reperiti nell'indagine

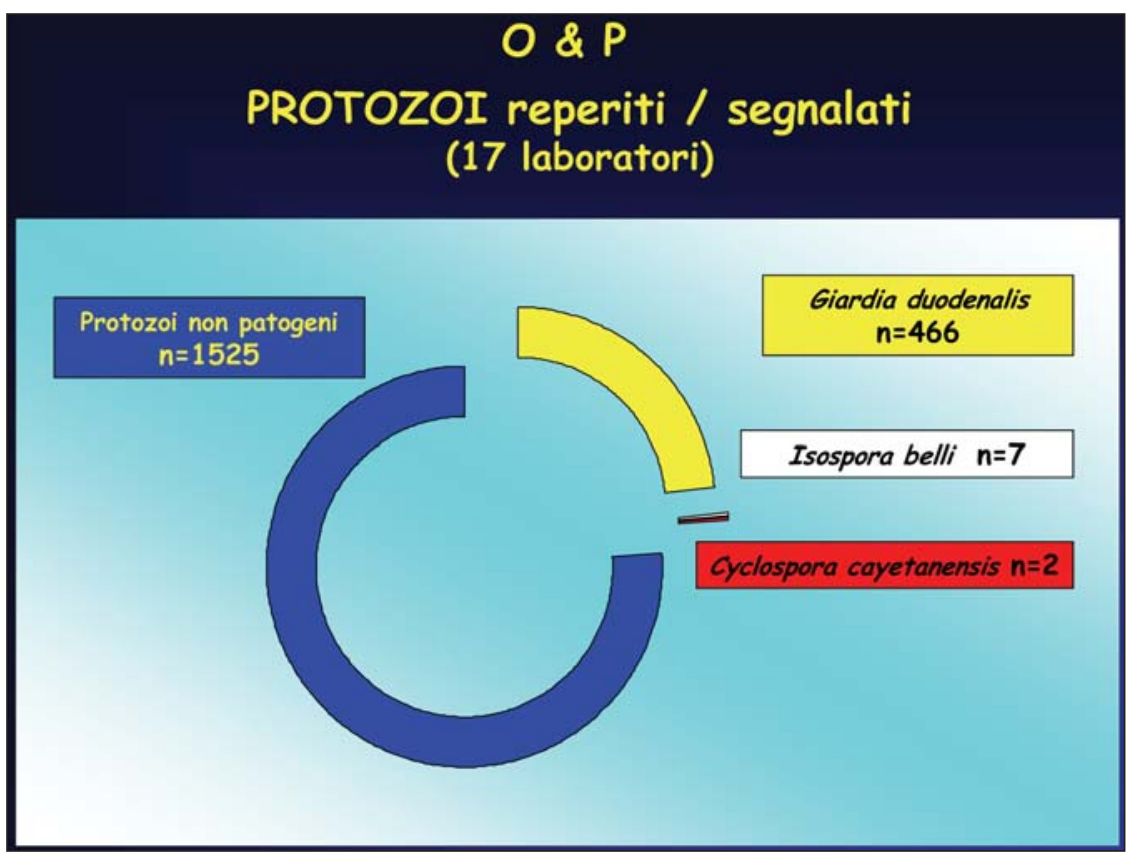

Tabella I I. Ricerca specifica mirata di E. vermicularis.

\begin{tabular}{clc}
\hline Eseguito in 22 laboratori & \multicolumn{2}{l}{ Scotch test: 22 laboratori } \\
\hline I campione solo & 6 laboratori & $27 \%$ \\
\hline $2-3$ campioni & I I laboratori & $50 \%$ \\
\hline Numero di campioni variabile (da I a 3 o più di 3) & 3 laboratori & $14 \%$ \\
\hline Non riferito & 2 laboratori & $9 \%$ \\
\hline
\end{tabular}

Tabella I2. Numero e percentuali di identificazione / ritrovamento di E. vermicularis nei vari Centri (22)

\begin{tabular}{|c|c|c|}
\hline \multicolumn{3}{|c|}{ Ricerca specifica (mirata) di Enterobius vermicularis (22 Centri) } \\
\hline Perugia & $16 / 73$ & $21.9 \%$ \\
\hline Pavia & $51 / 245$ & $20.8 \%$ \\
\hline Tione (TN) & $1 / 5$ & $20.0 \%$ \\
\hline Catania & $16 / 86$ & $18.6 \%$ \\
\hline Palermo & $127 / 782$ & $16.2 \%$ \\
\hline Milano & $48 / 322$ & $14.9 \%$ \\
\hline S. Bartolomeo in Galdo (BN) & $4 / 30$ & $13.3 \%$ \\
\hline Reggio Emilia & $7 / 54$ & $13.0 \%$ \\
\hline Padova & $15 / 121$ & $12.4 \%$ \\
\hline Iseo (BS) & $32 / 314$ & $10.2 \%$ \\
\hline Roma & $3 / 30$ & $10.0 \%$ \\
\hline Bergamo & $11 / 121$ & $9.1 \%$ \\
\hline Rho (MI) & $21 / 240$ & $8.8 \%$ \\
\hline Chivasso (TO) & $5 / 62$ & $8.1 \%$ \\
\hline Torino & $7 / 94$ & $7.4 \%$ \\
\hline Binasco (MI) & $7 / 100$ & $7.0 \%$ \\
\hline Gallarate (VA) & $9 / 131$ & $6.9 \%$ \\
\hline Verona & $3 / 56$ & $5.4 \%$ \\
\hline Montebelluna (TV) & $15 / 330$ & $4.4 \%$ \\
\hline Como & $20 / 454$ & $4.4 \%$ \\
\hline Napoli & $3 / 454$ & $4.3 \%$ \\
\hline Piancavallo (VB) & $0 / 12$ & - \\
\hline Complessivi & $401 / 3.277$ & $12.2 \%$ \\
\hline
\end{tabular}


Tabella 13.

Ricerca di S. stercoralis: $n^{\circ}$ campioni esaminati per soggetto.

\section{NUMERO DI CAMPIONI ESAMINATI PER SOGGETTO (12 laboratori)}

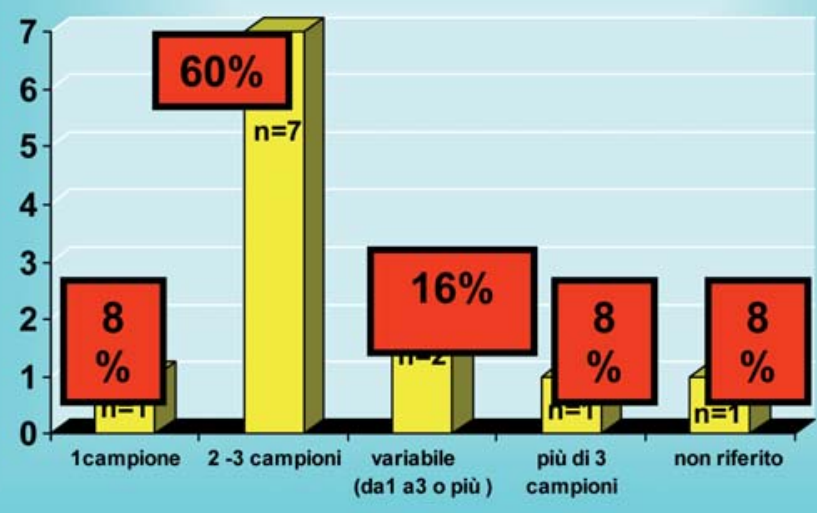

Tabella I4. Ricerca mirata di S. stercoralis (I 2 laboratori): i risultati.

\begin{tabular}{lcc}
\hline CENTRO LABORATORISTICO & S. stercoralis & S. stercoralis \\
\hline Roma & $\mathrm{n}^{\circ}$ positivi / $\mathrm{n}^{\circ}$ totale & \% ale di positività \\
\hline Catania & $2 / 6$ & $33.3 \%$ \\
\hline Bergamo & $3 / 10$ & $30.0 \%$ \\
\hline Iseo (BS) & $\mathrm{I} / 5$ & $20.0 \%$ \\
\hline Perugia & $2 / 14$ & $14.3 \%$ \\
\hline Torino & $\mathrm{I} / \mathrm{l} / 02$ & $10.8 \%$ \\
\hline Verona & $7 / 104$ & $6.7 \%$ \\
\hline Pavia & $4 / 107$ & $3.7 \%$ \\
\hline Padova & $109 / 4.884$ & $2.2 \%$ \\
\hline Como & $0 / 25$ & - \\
\hline Tione (TN) & $0 / 3$ & - \\
\hline S. Bartolomeo in Galdo (BN) & $0 / 1$ & - \\
\hline Complessivi & $0 / 5$ & - \\
\hline
\end{tabular}

Tabella I5. Ricerca mirata di D. fragilis e ricerca G. duodenalis a confronto (vedi anche testo)

\begin{tabular}{|c|c|c|c|c|c|}
\hline \multicolumn{6}{|c|}{ Ricerca mirata di D. fragilis e ricerca di G. duodenalis: confronto tra 7 laboratori } \\
\hline Laboratori & Col.ne & \multicolumn{2}{|c|}{ D. fragilis } & \multicolumn{2}{|c|}{ G. duodenalis } \\
\hline Bergamo & Giemsa & $1 / 6$ & $16.6 \%$ & $15 / 1.113$ & $1.3 \%$ \\
\hline Perugia & Giemsa & $87 / 1.289$ & $6.7 \%$ & $44 / 1.006$ & $4.3 \%$ \\
\hline Iseo, BS & Giemsa & $77 / 1.853$ & $4.2 \%$ & $4 \mathrm{I} / \mathrm{I} .853$ & $2.2 \%$ \\
\hline Padova & Giemsa & $48 / 2.517$ & $1.9 \%$ & \multicolumn{2}{|c|}{ NV } \\
\hline Montebelluna, TV & Giemsa & $63 / 6.785$ & $0.9 \%$ & $2 / 6.785$ & $0.1 \%$ \\
\hline S.Bartolomeo in Galdo, BN & Giemsa & $0 / 10$ & - & $1 / 120$ & $0.8 \%$ \\
\hline Pavia & Tricromica & $50 / 4.884$ & $1.0 \%$ & $85 / 4.884$ & $1.7 \%$ \\
\hline MEDIA & & \multicolumn{2}{|c|}{$326 / 17.344$} & \multicolumn{2}{|c|}{$|88 /| 5.76 \mid$} \\
\hline
\end{tabular}

Tabella I6. Ricerca mirata di Cryptosporidum spp (vedi anche testo).

\begin{tabular}{lcc}
\hline Pavia & $15 / 290$ & $5.2 \%$ \\
\hline Torino & $6 / 159$ & $3.8 \%$ \\
\hline Bergamo & $2 / 63$ & $3.2 \%$ \\
\hline Milano & $3 / 15 \mathrm{I}$ & $2.0 \%$ \\
\hline Catania & $\mathrm{I} / 50$ & $2.0 \%$ \\
\hline Como & $\mathrm{I} / 67$ & $1.5 \%$ \\
\hline Perugia & $\mathrm{I} / 84$ & $\mathrm{I} .2 \%$ \\
\hline Napoli & $\mathrm{I} / 210$ & $0.4 \%$ \\
\hline Altri 8 laboratori & $0 / 146$ & - \\
\hline Totale & $30 / 1.074$ & $2.8 \%$ \\
\hline
\end{tabular}


Tabella 17. Ricerca specifica di E. histolytica / E. dispar: è stata eseguita solo in 8 laboratori.

\begin{tabular}{lccc}
\hline Laboratorio & \multicolumn{1}{c}{ Metodo/i } & $N^{\circ}$ POS / N ${ }^{\circ}$ esaminati & $\%$ di positività \\
\hline Pavia & Tricromica,Coltura,Triage & $83 / 403$ & $20.6 \%$ \\
\hline Perugia & Tricromica & $10 / 62$ & $16.1 \%$ \\
\hline Torino & Tricromica,Coltura,Triage & $9 / 61$ & $14.7 \%$ \\
\hline Bergamo & Triage & $4 / 15 I$ & $2.6 \%$ \\
\hline Catania & Tricromica & $5 / 1.060$ & $0.5 \%$ \\
\hline Reggio Emilia & Triage & $3 / 1.916$ & $0.2 \%$ \\
\hline Altri 2 Centri & vari & $0 / 27$ & - \\
\hline TOTALE & & $1 / 4 / 3.680$ & $3.1 \%$ \\
\hline
\end{tabular}

\section{BIBLIOGRAFIA}

1. Barbaro GB, Cavallino AM, Gianotti A, Patrone C, Robello A. Esami coproparassitologici: analisi di una casistica quinquennale raccolta nel territorio del Ponente Genovese. GIMMOC 2001; V (1): 18-20.

2. Bernieri F, Casella P, Crotti D, et al. Linee Guida Operative per la diagnosi delle Parassitosi Intestinali. Microbiol Med 2005; 20 (I): 39-46.

3. Bernieri F, Galli D, Viti F, et al. Parassitosi enteriche autoctone. Microbiol Med 1994; 9 (3): 415-8.

4. Bernieri F, Galli D, Crotti D, Del Sante M. Studio Policentrico dell'Associazione Microbiologi Clinici Italiani - Comitato di Studio di Parassitologia (AMCLI-CoSP) sulla diffusione delle parassitosi intestinali in Italia: parassitosi intestinali nell'immigrato. Giorn It Med Trop 1996; 1 (3-4): 97-9.

5. Brunelli T, Degl'Innocenti R, Pellegrini L, et al. Parassitosi intestinali umane nell'area di Prato (20082011). Poster, XLI Congresso Nazionale AMCLI, 1316 novembre 2012, Rimini.

6. Crotti D. Le parassitosi intestinali ed uro-genitali. Caleidoscopio Italiano, Medical System SpA, ISSN 0394 3291; 2005.

7. Crotti D. Enterobiasis in Italy today: a Primary School's experience. Microbiol Med 2008; 23 (I): 647.

8. Crotti D, Bernieri F, AMCLI-CoSP. Prospettive diagnostiche nelle parassitosi intestinali. Microbiol Med 1997; 12 (2): 74-8

9. Crotti D, Bernieri F, Raglio A, Galli D. L'esame coproparassitologico: indicazioni di base per una adeguata diagnostica. SIMG 2005; 5/6: 28-32.

10. Crotti D, Crotti S, Sensi M, et al. Dientamoeba fragilis detection in suid populations: an emerging zoonosis hypothesized in Central Italy. Microbiol Med 2012; 27 (3): 137-40.

11. Crotti D, D’Annibale ML. Parassitosi intestinali autoctone: ruolo di Dientamoeba fragilis nelle infezioni umane. Ann Ig 2007; 19: 27-34.

12. Crotti D, D'Annibale ML, Basileo M, La Torre G. Sondaggio preliminare copro-parassitologico in una zona andina del Perù. Infez Med 2007; 3: 181-6.

13. Crotti D, D'Annibale ML, Crotti S. Opisthorchiasi autoctona al Lago Trasimeno (Perugia): descrizione di due episodi epidemici da Opisthorchis felineus e problematiche diagnostiche differenziali. Microbiol Med 2007; 22 (I): 36-41.

14. Crotti D, D’Annibale ML, Fonzo G, Lalle M, Cacciò SM, Pozio E. Dientamoeba fragilis is more prevalent than Giardia duodenalis in children and adults attending a day care centre in Central Italy. Parasite 2005; 12: $165-70$.

15. Crotti D, D’Annibale ML, Rossi S. Strongyloides stercoralis e strongyloidiasi in Umbria: analisi di una casistica decennale. Microbiol Med 2005; 20 (4): 250-6.

16. Crotti D, Raglio A, Galli D, Bernieri F. L'esame copro parassitologico nei bambini. Indicazioni e indagini raccomandate. Quaderni acp 2004; 12 (1): 39-42.

17. D’Annibale ML. Infestazione spuria da Dicrocoelium dendriticum. Microbiol Med 2004; 399-400.

18. D’Annibale ML, Bracciale S, Vitali M, Fonzo G, Verdini C, Papili R. Parasitic intestinal infection in humans between 2006 and 2007. Microbiol Med 2009; 24 (I): 19-24.

19. D’Annibale ML, Crotti D. Parassitosi intestinali umane nel Perugino nel corso del 2005. Microbiol Med 2007; 22 (I): 57-65.

20. Ferlini M, Calia F, Alfano AM, et al. Caso clinico di occlusione intestinale in soggetto con doppia infestazione da Ascaris lumbricoides e Trichuris trichiura. Microbiol Med 1999; 14 (4): 343-4.

21. Fletcher SM, Stark D, Harkness J, Ellis J. Enteric Protozoa in the Developed World: a Public Health Perspectives CMC 2012; 25 (3): 420-49.

22. Gigli C, Crobu MGS, Ferrante R, Cestagalli L, Fortina G. Indagine epidemiologica 2000-2002 dei parassiti fecali nell'area novarese. Microbiol Med 2003; 18 (2): 188.

23. Giordano S, Troia G, Miraglia P, Scarlata F. Epidemiologia delle parassitosi intestinali in Sicilia occidentale. Osservazioni relative agli anni 19932000. Infez Med 2001; 3: 154-7.

24. Grande R, Ranzi ML, Restelli A, Maraschini A, Perego L, Torresani E. Prevalenza delle parassitosi intestinali nella popolazione afferente alla Fondazione IRCCS Cà Granda, Ospedale Maggiore Policlinico di Milano: confronto tra il 1984-1985 e il triennio 20072009. Infez Med 2011; 1: 28-38.

25. Guidetti C, Capatti C, Ricci L, Vecchia L. Intetsinal parasitosis in Reggio Emilia during 2007: critical analysis of results. Microbiol Med 2009; 24 (2): 103 7.

26. Guidetti C, Ricci L, Vecchia L. Prevalenza delle parassitosi intestinali a Reggio Emilia e provincia nel corso del 2009. Infez Med 2010; 3: 154-61.

27. Guidetti C, Ricci L, Vecchia L. Eziologia delle parassitosi intestinali in un campione di studenti mozambicani: breve indagine conoscitiva. Infez Med 2011; 3: 157-65.

28. Guidi C, D'Annibale ML, Crotti D. Un caso di infestazione da Diphyllobothrium latum in Umbria, Italia centrale. GIMMOC 2005; IX (3): 172-5.

29. Magi B, Frati E, Bernini L, Sansoni A, Zanelli G. Dicrocoelium dendriticum: a true infection? Infez Med 2009; 2: 115-6.

30. Mangoni P, Ferrari L, Mazzei G, Bernieri F. Strongyloidiasis in Po valley: serological and parasitological screening in blood donors of Cremona. 
Microbiol Med 2012; 27 (3): 133-6.

31. Marangi M, Zechini B, Fileti A, Quaranta TG, Aceti A. Hymenolepis diminuta infection in a child living in the urban area of Roma, Italy. J Clin Microbiol 2003; 41 (8): 3994-5.

32. Nocera E, Bozzelli L, Gallo MA, et al. Epidemiologia delle parassitosi intestinali in una popolazione di immigrati Extracomunitari. Microbiol Med 2006; 21 (4): 328-32.

33. Pernice L, Forino D, Leonaldi R, Lo Giudice L, Costa AM, Ioli A. Indagini parassitologiche condotte dal Servizio di Parassitologia dell'Università di Messina nel periodo 1996-2003. Riv Parassitol 2004; XXI (LXV) - N. 2: 119-28.
34. Pica R. Indagine di laboratorio sulle parassitosi: esperienza dell'Ospedale San Giovanni di Roma. Parassitologia 1998; 40 (Suppl. 1): 134.

35. Pica R. Emerging intestinal infections: the heterophyids. Parassitologia 2002; 44 (Suppl. 1): 135.

36. Pozio E. Epidemiology and control prospects of foodborne parasitic zoonoses in the European Union. Parassitologia 2008; 50: 17-24.

37. Scarlata F, Giordano S, Infurnari L, et al. Un caso di addome acuto da infestazione massiva da ascaridi. Infez Med 2008; 1: 37-9.

38. Tan KSW. New insights on classification, identification, and clinical relevance of Blastocystis spp. CMR 2008; 21 (4): 639-65. 